\title{
Functional impairment in adolescents and young adults with emerging mood disorders
}

Jan Scott, Elizabeth M. Scott, Daniel F. Hermens, Sharon L. Naismith, Adam J. Guastella, Django White, Bradley Whitwell, Jim Lagopoulos and Ian B. Hickie

\section{Background}

Between 30 and $60 \%$ of adults with unipolar or bipolar disorders exhibit impairments across multiple domains. However, little is known about impaired functioning in youth with mood disorders.

\section{Aims}

To examine the prevalence of objective, subjective and observer-rated disability in a large, representative sample of young people with a primary mood disorder.

\section{Method}

Individuals aged 16-25 years presenting to youth mental health services for the first time with a primary mood disorder participated in a systematic diagnostic and clinical assessment. Impairment was assessed using objective (unemployment or disability payments), observer- (Social and Occupational Functioning Assessment Scale; SOFAS) and selfrated measures (role functioning according to the Brief Disability Questionnaire).

\section{Results}

Of 1241 participants (83\% unipolar; $56 \%$ female), at least 30\% were functionally impaired on the objective, self-rated and/or observer-rated measures, with $16 \%$ impaired according to all three criteria. Even when current distress levels were taken into account, daily use of cannabis and/or nicotine were significantly associated with impairment, with odds ratios (OR) ranging from about 1.5 to 3.0. Comorbid anxiety disorders were related to lower SOFAS scores $(\mathrm{OR}=2-5)$.

\section{Conclusions}

Levels of disability were significant, even in those presenting for mental healthcare for the first time. Functional impairment did not differ between unipolar and bipolar cases, but some evidence suggested that females with bipolar disorder were particularly disabled. The prevalence of comorbid disorders (50\%) and polysubstance use (28\%) and their association with disability indicate that more meaningful indicators of mood episode outcomes should focus on functional rather than symptom-specific measures. The association between functioning and nicotine use requires further exploration.

\section{Declaration of interest}

I.B.H. has led projects for health professionals and the community supported by governmental, community agency and pharmaceutical industry partners (Wyeth, Eli Lily, Servier, Pfizer, Astrazeneca) for the identification and management of depression and anxiety. He has received honoraria for educational seminars supported by the pharmaceutical industry (including Pfizer, Servier and Astra Zeneca). He has served on advisory boards convened by the pharmaceutical industry (including Bristol-Myers Squibb, Eli Lilly and Pfizer) in relation to specific antidepressants. His research has been supported in part by pharmaceutical manufacturers (including Servier and Pfizer). D.F.H. has received honoraria for educational seminars from Janssen-Cilag. E.M.S. has received honoraria for educational seminars related to the clinical management of depressive disorders supported by Servier and Eli Lilly. She has participated in an advisory board for Pfizer. J.S.'s research has been supported by funds provided to the NTW NHS Trust by Astra-Zeneca and Janssen-Cilag for investigator-initiated studies on medication adherence. She has received honoraria for educational seminars supported by the pharmaceutical industry and has served on advisory boards convened by the pharmaceutical industry (including Astra-Zeneca, Janssen-Cilag, Lundbeck, Pfizer, Sanofi-Aventis and Servier).
Between 30 and $60 \%$ of adults with a unipolar or bipolar disorder report significant impairments in social and occupational functioning, including an inability to return to pre-illness social or professional roles, increased number of days absent from work per annum and increased rates of unemployment, disability benefit payments, medical retirements and difficulties performing daily living tasks. ${ }^{1-10}$ The clinical, social and economic consequences of these disabilities have reinforced the need to promote effective interventions in the earliest phases of illness, ${ }^{10-13}$ as it is assumed that youth presenting with recent-onset mood disorders will exhibit fewer and less severe impairments, and that these should be more amenable to modification. ${ }^{11-13}$ However, in contrast to data on older adults with established mood disorders, there is little research on the prevalence and factors associated with social disability in representative samples of adolescents and young adults with mood disorders. The studies available are limited by small sample sizes and/or recruitment biases. ${ }^{14-20}$ For example, half of the studies recruited attendees at specialist affective disorders programmes, ${ }^{15,17,19}$ whereas others examined community samples of individuals who met diagnostic criteria for unipolar or bipolar disorder but who were neither distressed by their symptoms nor help-seeking. ${ }^{16,18}$ Despite the heterogeneity in sampling, the findings do suggest that even young adults with mood disorders already exhibit signs of impairment. However, there is conflicting evidence regarding the extent to which functioning is influenced by mood disorder subtype (unipolar or bipolar disorder), comorbidities and other demographic or illness characteristics. ${ }^{15-17}$

Given the apparent gap in the understanding of functional impairment in young people with emerging mood disorders, this study examines the prevalence of objective, subjective and observer-rated disability in a large, representative sample of young people who presented to youth mental health services with a primary diagnosis of either unipolar or bipolar disorder. It also explores factors that are significantly associated with these different levels of disability. 


\section{Method}

The study was approved by the University of Sydney Human Research Ethics Committee. All participants aged 18 years or more gave independent, written informed consent; additional parental consent was obtained for individuals aged $<18$ years. Study participants were drawn from a larger cohort of consecutive referrals to youth mental health clinics in and around Sydney who were recruited to a case register of young people with mood, psychotic, developmental and other mental disorders between October 2007 and January 2012. ${ }^{12,20}$ Individuals were included in the present study on the basis of the following inclusion criteria: (a) having a clinical diagnosis of unipolar or bipolar disorder (type I or II); (b) being aged 16-25 years at the time of initial assessment (this age range was chosen as it is the peak onset period for severe mood disorders and $\geqslant 16$ years is the age at which individuals qualify for financial assistance and/or disability payments in Australia); and (c) being willing and able to give written informed consent (and/or parental consent was obtained). Exclusion criteria were medical instability (as determined by a psychiatrist), lack of capacity to give informed consent, history of neurological disease (such as a tumour, head trauma, epilepsy), medical illness known to have an impact on cognitive and brain function (such as cancer) and a clinically assessed IQ below 70, and/or insufficient English to participate in the research protocol.

\section{Intake assessment}

A structured clinical assessment was undertaken and key referral information and other selected details of clinical history were recorded on a specifically designed proforma. ${ }^{12,20}$ In order to improve the quality of information on diagnoses and comorbidities collected at the clinic (especially clarifying the difference between subthreshold and threshold major depression), the Brain and Mind Institute utilises three additional approaches. First, they monitor interrater reliability; second, 'caseness' is reassessed at least once over the following 4 weeks; and third, the cases of any individuals where diagnoses or assessments are unclear are discussed at a consensus meeting. In a substudy of $>200$ individuals, the rate of agreement between the treating clinician (using the initial proforma) and the independent consensus raters was high (kappa $(\kappa)=0.72, P<0.001)$. Of the discordant ratings, $>80 \%$ were assigned to subthreshold caseness (and hence were excluded from our study as those individuals did not meet diagnostic criteria). ${ }^{21}$ This study utilised case register data on primary and secondary DSM-IV diagnoses ${ }^{22}$ and information on the prevalence and degree of alcohol and substance use and misuse (including nicotine, cannabis, amphetamine-type stimulants, and/or other substances) for the previous 3 months as rated on the Alcohol, Smoking and Substance Involvement Screening Test (ASSIST). ${ }^{23}$

At initial assessment, participants completed the Kessler-10 (K10) questionnaire, ${ }^{24}$ which is a well-validated measure of general symptoms and distress (such as 'How often do you feel so nervous that nothing can calm you down?'; 'How often do you feel that everything is an effort?'). Each K10 question is rated on a 1-5 scale (from none of the time to all of the time) and the total score ranges from 10 to 50 , with scores of 10-19 suggesting the individual is likely to be 'well', and higher scores indicating higher levels of distress. A score $>30$ is typical of severe mental disorders in the community. ${ }^{24,25}$ This measure was used instead of separate ratings of manic or depressive symptoms because perceived distress (associated with acute symptoms or dysfunction) rather than diagnosis per se is the commonest reason for help-seeking in young people. ${ }^{26,27}$ Also, the questionnaire has been widely used and applied to a range of diagnoses and clinical presentations in adult as well as adolescent populations in clinical and community settings.

\section{Social functioning and disability}

The prediction of functional level was examined from three perspectives using an external/independent marker of disability, and observer and self-report ratings. These measures capture different aspects of disability or impairment and reflect the most widely used assessments in previous research on functioning in a range of diagnostic and demographic groups.

\section{Receipt of financial support}

Participants were asked to indicate if they were in education (student at a secondary or tertiary education establishment), training or employment on a full-time $(\geqslant 35 \mathrm{~h})$ or part-time basis. If they were identified as 'vocationally inactive', they were also asked to indicate whether they received financial assistance from the government (unemployment benefits, disability support pension or other state benefits).

\section{Social and Occupational Functioning Assessment Scale}

An observer-rated measure, the Social and Occupational Functioning Assessment Scale (SOFAS), ${ }^{28}$ assesses functioning on a 0-100 scale, with lower scores suggesting more severe impairment. The instructions for completion highlight that the rater should endeavour to avoid confounding the rating with clinical symptoms.

\section{Brief Disability Questionnaire}

As in previous studies, two items from the Brief Disability Questionnaire (BDQ) ${ }^{29}$ were used that focus on subjective ratings of 'role impairment' over the previous month. Participants reported how many days they were unable to complete their normal activities (BDQ-Impaired: 'days unable to complete activities' per month) or how many days they were unable to get out of bed (BDQ-Unable: 'days in bed' per month).

\section{Statistical analysis}

Statistical analyses were performed using SPSS 20.0 for Windows. Missing data analysis suggested a random pattern to the small amount of information lost; therefore the planned analyses excluded participants with missing items list-wise (hence numbers included may vary by analysis). As $84 \%$ of those with bipolar disorder met diagnostic criteria for bipolar II disorder, all participants with bipolar disorder were included within one group for all subsequent analyses. For continuous variables such as age at presentation, SOFAS, K10, BDQ-Impaired, BDQ-Unable we report means, standard deviations (s.d.) and medians (when relevant).

Four groups were identified according to diagnostic subtype and gender (female bipolar; female unipolar; male bipolar; male unipolar groups) and differences were examined in demographic, clinical and functional factors and variables using chi-squared tests or analysis of variance (with Dunnett's post hoc t-tests used to examine subgroup differences). As the BDQ item scores showed skewed non-normal distributions they were log transformed prior to ANOVA. Participants with missing data were excluded list-wise from the analyses. All statistical tests were 2-tailed and used a significance level of $\alpha=0.05$. 
To explore factors associated with disability and functional impairment, we used backward stepwise logistic regression (BSLR) for analyses of membership of two subgroups and multinomial logistic regression (MNLR) for analyses of three or more subgroups. Disability was defined by receipt of financial support (yes/no), whereas BDQ data were used as subjective markers of functional impairment. As 'BDQ-Impaired' and 'BDQ-Unable' ratings are highly interrelated and statistically showed a significant correlation $(r=0.48)$ we chose BDQ-Unable for the BSLR analysis (with participants allocated to groups according to whether the number of days per month they were unable to get out of bed was above or below the sample median). For both BSLR analyses, $\alpha$ levels for entry and exclusion were set at $P=0.15$ and based on the likelihood ratio statistic. MNLR was used to analyse factors associated with observer-rated impairment in functioning (SOFAS score). Participants were categorised into one of four subgroups according to their SOFAS score (with boundaries between groups defined by the 25th, 50th, 75th quartile scores): severely impaired (score $<51$ ), moderately impaired (score 51-60), impaired (score 61-65) and mildly impaired (score $>65$ ). The group with the highest level of functioning (mildly impaired) was selected as the reference category and the forward (likelihood ratio) procedure was used for the regression. Variables were entered into the model if they had a significance level of $P<0.050$. The same factors and variables were included in each of the BSLR and MNLR models: age, gender, mood disorder subtype (bipolar $v$. unipolar disorder) and K10 score, followed by the ASSIST ratings of nicotine, cannabis and alcohol use/misuse (five-point scale: never, 1-2 occasions, monthly, weekly, daily or almost daily), and presence or absence of comorbidities (none, behavioural/developmental disorders, anxiety disorders, other). Factors associated with subgroup membership are reported, including odds ratios (ORs) and the $95 \%$ confidence intervals.

Finally, an exploratory BSLR analysis was undertaken comparing two selected subgroups of participants: those categorised as impaired on all three measures of functioning (receiving financial assistance, BDQ-Unable $>$ median; SOFAS score $<$ median) compared with the subgroup who were not categorised as impaired on any of the measures. The independent variables entered into the analysis comprised the significant associations identified in one or more of the separate regression models.

\section{Results}

\section{Sample characteristics}

From the case register of 2607 individuals (with a wide range of clinical diagnoses and ages at presentation), 1795 were identified with a probable primary diagnosis of mood disorder. From that subsample, 1241 individuals met diagnostic caseness for a unipolar or bipolar disorder and fulfilled the study eligibility criteria.

As shown in Table 1, the sample mean age was 19.4 years (s.d. $=2.61$; median, 19); there were $701(56 \%)$ females and 540 (44\%) males. The majority $(54 \%, n=670)$ were living with family members, but more than a third were living independently $(28 \%$ in shared accommodation, 7\% alone). Over 1000 participants were classified as having unipolar disorders (83\%); 30 individuals (15 unipolar, 15 bipolar disorder) had a history of psychotic symptoms. The mean K10 score was 30.86 (s.d.=8.29; median, 32) suggesting high levels of psychological distress. Further analysis identified that females with unipolar or bipolar disorder had significantly higher scores than males with bipolar disorder $(F=$ 10.17 , d.f. $=3,1003, P=0.001)$. Comorbid DSM-IV disorders were reported in $51 \%(n=631)$ of participants; anxiety disorders were the most frequent $(n=312,25 \%)$, whereas behavioural and development disorders (such as autistic spectrum and attentiondeficit hyperactivity disorders) were relatively uncommon $(n=73,6 \%) ; 20 \%$ of the sample $(n=246)$ reported one or more other comorbidities. The distribution of comorbidities showed significant differences according to gender and/or diagnosis $\left(\chi^{2}=67.1\right.$, d.f. $\left.=12, P=0.001\right)$ : females with unipolar disorder (30\%) were significantly more likely than other subgroups to have 'any anxiety disorder' and 'any behavioural or developmental disorders' was four times more prevalent in bipolar compared with unipolar disorder (17\% bipolar, $4 \%$ unipolar disorder).

Table 1 also demonstrates that one in four participants (208/ 925) reported that they never used alcohol, but 7\% (68/925) reported daily use. For nicotine, the comparable rates were: no use $49 \%(451 / 930)$ and daily use 35\% (327/930). For cannabis, $68 \%$ reported no use (618/911), and 7\% were daily users (67/ 911); amphetamine use was uncommon (reported by 15\%) and only three individuals $(0.3 \%)$ were daily users. Overall, just over $20 \%$ of participants reported that they had never used alcohol or any other substance $(n=201 / 901)$, whereas $252(28 \%)$ reported concurrent use of alcohol, nicotine and cannabis. Using the fivepoint rating (never to daily), there were significant differences in use of nicotine $\left(\chi^{2}=25.88\right.$; d.f. $\left.=2, P=0.011\right)$ and cannabis $\left(\chi^{2}=22.89\right.$, d.f. $\left.=12, P=0.029\right)$ : daily nicotine use was highest in females with bipolar disorder $(44 \%)$, whereas daily cannabis use was twice as frequent in males compared with females $(11 \%$ v. $5 \%)$.

\section{Table 1 Sample characteristics including demography,} diagnoses, psychological distress and disability measures ${ }^{a}$ Characteristic

\begin{tabular}{|c|c|c|}
\hline Age, years: mean (s.d.) & $19.4(2.61)$ & 1241 \\
\hline Gender & & 1241 \\
\hline Male, \% (n) & $44(540)$ & \\
\hline Female, \% ( $n)$ & $56(701)$ & \\
\hline Living with family members & $54(670)$ & 1241 \\
\hline Mood disorder subtype & & 1241 \\
\hline Unipolar disorder, \% (n) & $83(1036)$ & \\
\hline Bipolar disorder, \% (n) & $17(205)$ & \\
\hline Gender diagnosis & & 1241 \\
\hline Male bipolar, \% (n) & 7 (92) & \\
\hline Male unipolar, \% (n) & $36(448)$ & \\
\hline Female bipolar, \% (n) & 9 (113) & \\
\hline Female unipolar, \% (n) & $47(588)$ & 1241 \\
\hline Kessler-10 score, mean (s.d.) & $30.86(8.29)$ & \\
\hline Comorbid disorder & & 1241 \\
\hline None, \% (n) & $49(610)$ & \\
\hline Any anxiety disorder, \% ( $n)$ & $25(312)$ & \\
\hline Any behavioural or developmental disorder, \% (n) & $6(72)$ & \\
\hline Any other comorbidity, \% (n) & $20(246)$ & \\
\hline Alcohol and substance use, \% never $v$. \% daily & & \\
\hline Alcohol & 22 v. 7 & 925 \\
\hline Nicotine & 48 v. 35 & 930 \\
\hline Cannabis & 68 v. 7 & 911 \\
\hline Amphetamines & 85 v. 0.3 & 901 \\
\hline Financial support, \% (n) & $36(272)$ & 750 \\
\hline Days per month BDQ-Impaired, mean (s.d.) & $9.43(9.38)$ & 784 \\
\hline Days per month BDQ-Unable, mean (s.d.) & $4.72(6.86)$ & 813 \\
\hline SOFAS score, mean (s.d.) & $58.82(12.15)$ & 1241 \\
\hline \multicolumn{3}{|c|}{$\begin{array}{l}\text { a. The percentages are rounded to nearest whole numbers. Kessler- } 10 \text { Questionnaire } \\
\text { (higher scores; more distressed); Brief Disability Questionnaire (BDQ; higher scores, } \\
\text { more impaired) and Social \& Occupational Functioning Assessment Scale (SOFAS; } \\
\text { higher scores, better functioning). }\end{array}$} \\
\hline
\end{tabular}




\section{Prevalence of impairment}

In total, $36 \%$ of the sample was receiving some type of financial assistance or state benefits, with $6 \%$ of individuals (64/1047 with data available) already on a disability support pension specifically because of their mental illness. About 800 individuals completed the BDQ self-ratings of impairment; the mean number of days per month with reduced functioning was 9.43 for BDQ-Impaired (s.d. =9.38; median, 7) and 4.72 for BDQ-Unable (s.d.=6.86; median, 2). The mean SOFAS score was 59 (s.d. $=12.15$; median, 60 ) indicating at least moderate social and occupational impairment. As shown in Fig. 1, the mean number of days per month for BDQ-Unable was significantly higher in females with bipolar disorder than other groups $(F=2.99$, d.f. $=3,809$, $P=0.03$ ), but other parameters of impairment did not show significant between-group differences.

\section{Factors associated with impairment}

The BSLR model for receipt of financial support correctly classified $66 \%$ of participants' cases $\left(\chi^{2}=39.33\right.$, d.f. $=7$, $P=0.001, n=750)$. As shown in Table 2, the best combination of independent factors related to impairment was: older age $(\mathrm{OR}=1.12, \quad 95 \%$ CI $1.04-1.21, \quad P=0.002)$, female gender $(\mathrm{OR}=1.47,95 \% \mathrm{CI} 1.09-2.33, P=0.039)$, and (separately) daily nicotine use $(\mathrm{OR}=1.61,95 \% \mathrm{CI} 1.02-2.55, P=0.042)$ and daily cannabis use $(\mathrm{OR}=2.49,95 \% \mathrm{CI} 1.16-5.39, P=0.001)$.

When impairment was defined subjectively by how many days per month the individual reported that they were unable to get out of bed, BSLR correctly classified $64 \%$ of participants' cases $\left(\chi^{2}=100.55\right.$, d.f. $\left.=7, P=0.01, n=816\right)$. As noted in Table 2, the combination of variables associated with greater level of subjective impairment (BDQ-Unable score above the sample median) was: female gender $(\mathrm{OR}=1.07,95 \% \mathrm{CI} 1.05-1.09, P=0.007)$; K10 score $(\mathrm{OR}=1.08,95 \%$ CI $1.05-1.09, P=0.001)$ and daily cannabis use $(\mathrm{OR}=2.46,95 \%$ CI 1.35-4.49). Further exploration revealed that cannabis use showed a 'dose-response'-type pattern with
ORs of 1.25 (95\% CI 1.10-1.41, P=0.001) for any reported use, 1.56 (95\% CI 1.00-2.42) for occasional use and 2.29 (95\% CI 1.23-4.25) for at least monthly use. Mood disorder subtype just failed to reach statistical significance in this model (bipolar $v$. unipolar $\mathrm{OR}=1.47,95 \%$ CI $0.98-2.21, P=0.062$ ).

The MNLR model for the four SOFAS subgroups significantly differentiated the participants that were least impaired (SOFAS score $>65)$ from the other groups $\left(\chi^{2}=143.85\right.$, d.f. $=60$; $P=0.0001, n=857)$. As shown in Table 3 , individuals with the lowest SOFAS score $(<51)$ were significantly more likely to have a higher $\mathrm{K} 10$ score $(\mathrm{OR}=1.08,95 \%$ CI $1.06-1.11, P=0.001)$, a comorbid anxiety disorder $(\mathrm{OR}=5.85,95 \%$ CI 1.98-17.27, $P=0.001)$ and a daily nicotine habit $(\mathrm{OR}=1.21,95 \% \mathrm{CI} 1.05-$ 1.37) compared with the mildly impaired group. Those with a SOFAS score of 51-60 were significantly more likely than the mildly impaired group to be female $(\mathrm{OR}=1.36,95 \%$ CI $1.05-1.96$, $P=0.032)$ and have a higher $\mathrm{K} 10$ score $(\mathrm{OR}=1.07,95 \%$ CI 1.04 $1.11, P=0.001)$. Those with a SOFAS score of $60-65$ had a higher K10 score $(\mathrm{OR}=1.03,95 \%$ CI $1.00-1.05, P=0.02)$ and higher prevalence of comorbid anxiety disorders $(\mathrm{OR}=2.09,95 \% \mathrm{CI}$ $1.17-3.73, P=0.002)$ than the mildly impaired group.

Overall, $16 \%$ individuals met all three criteria for impairment, whereas $17 \%$ met none of the criteria for impairment. When an exploratory BSLR was undertaken comparing the subgroups of participants categorised as having poor functioning on all three impairment measures $(n=115) v$. those categorised as having good functioning on all three measures $(n=125)$, two factors correctly classified $75 \%$ of all participants $\left(\chi^{2}=42.51\right.$, d.f. $\left.=7, P=0.01\right)$ : high K10 score $(\mathrm{OR}=1.14,95 \%$ CI $1.09-1.20, P=0.001)$ and daily nicotine use $(\mathrm{OR}=3.18,95 \% \mathrm{CI} 1.54-6.56, P=0.002)$. Gender just failed to reach statistical significance in this comparison of selected subgroups, but it was noteworthy that females with bipolar disorder $(12 / 20,60 \%)$ were more likely to be categorised as impaired on all three ratings than females with unipolar $(53 / 99,54 \%)$, males with unipolar $(43 / 100,43 \%)$ or males with bipolar disorder (7/21, 33\%).

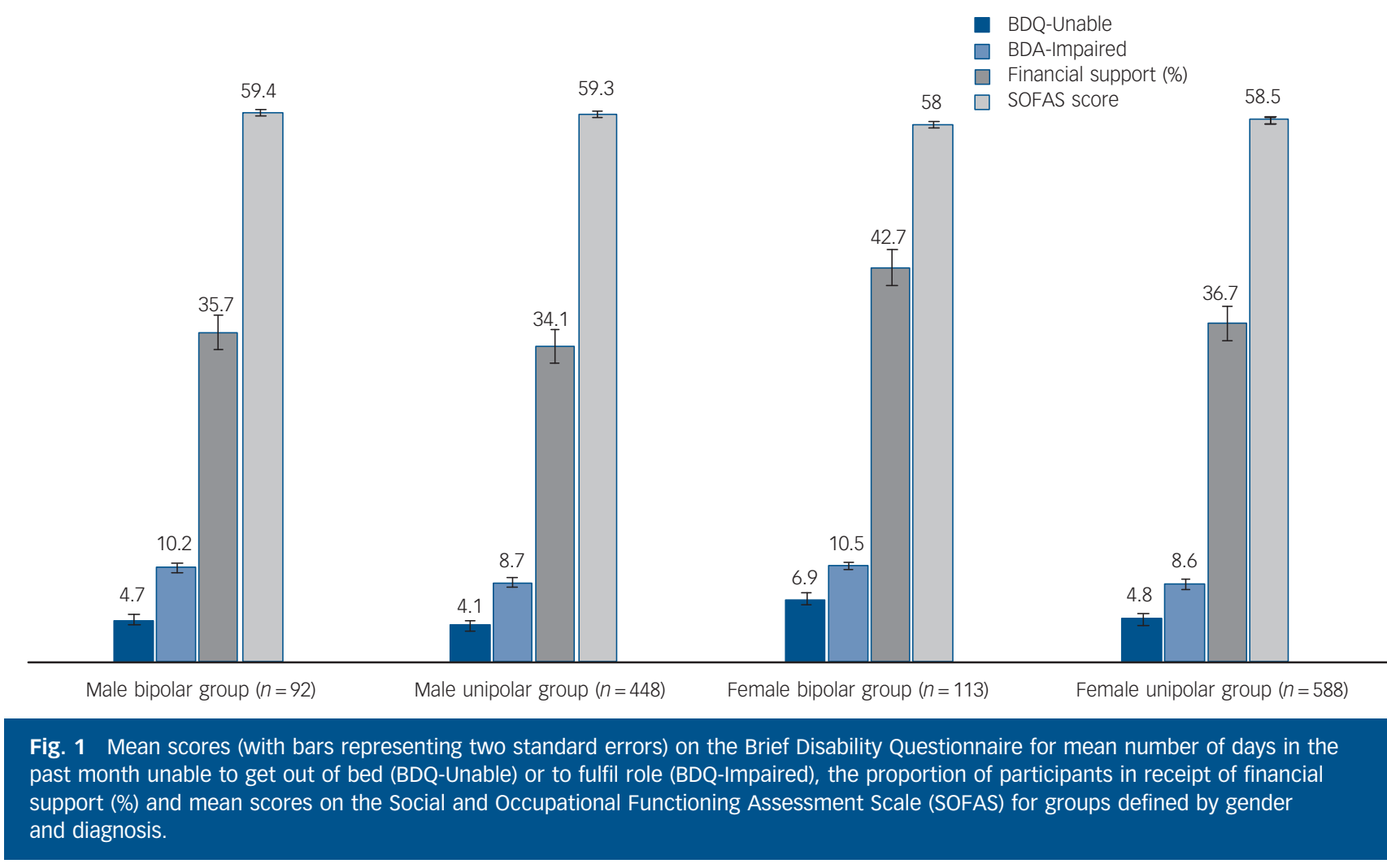


Table 2 Backward stepwise logistic regression analyses demonstrating the factors most strongly related to objectively measured disability (receipt of financial support $v$. no financial support) and to self-rated role impairment (Brief Disability Questionnaire (BDQ)-Unable $>2$ days in bed per month $v . \leqslant 2$ days)

OR $(95 \% \mathrm{Cl})$

\begin{tabular}{ll} 
Factors associated with financial support & \\
Older age & $1.12(1.04-1.21)$ \\
Female gender & $1.47(1.09-2.33)$ \\
Daily nicotine use & $1.61(1.02-2.55)$ \\
Daily cannabis use & $2.49(1.16-5.39)$ \\
\hline Factors associated with days spent in bed per month & \\
Female gender & $1.07(1.05-1.09)$ \\
Kessler-10 score & $1.08(1.05-1.09)$ \\
Daily cannabis use & $2.46(1.35-4.49)$
\end{tabular}

\section{Discussion}

\section{Main findings}

To our knowledge, this is the largest study undertaken that examines levels of disability and functional impairment in a representative clinical sample of young people presenting for the first time to mental health services with a DSM-IV diagnosis of unipolar or bipolar disorder. To try to give some context to our findings on disability and associated factors, it is noteworthy that the Young Australian Health and Well-being Survey of 2011 identified that about $11-15 \%$ of 16 - to 25 -year-olds were receiving financial support, about $9 \%$ scored $\geqslant 30$ on the K10 scale, $7 \%$ met criteria for harmful rates of alcohol intake, about $20 \%$ reported daily nicotine use and about 3\% reported harmful use of cannabis. ${ }^{30}$ As shown by our findings, the problems reported in our clinical sample significantly exceed all of these rates.

As might be expected in a cohort of 16- to 25-year-olds with recent-onset mood disorders, the majority of participants presented with a unipolar profile and only a minority of individuals with bipolar disorder met criteria for bipolar I disorder. This difference also emphasises that depression in this age group is often a 'forerunner' of later bipolar disorder or psychosis; alternatively some of the cohort may never experience a further mood disorder episode. However, the possibility of future diagnostic uncertainty is less important than the finding that the prevalence and patterns of comorbidities, alcohol and/or substance use in this cohort mirror those seen in general adult psychiatry out-patients with long-established mood disorders, ${ }^{3-8,31}$ suggesting that these problems are present from the earliest phases of these disorders. In this youth sample, half the participants reported at least one comorbid mental disorder, and polysubstance use occurred in about a third. Interestingly, nicotine and cannabis were used in preference to alcohol, with evidence that females with bipolar disorder favoured daily nicotine use, whereas males with unipolar and bipolar disorder preferred daily cannabis use.
An examination of the objective, subjective and observer-rated measures of disability indicate that about $16 \%$ of participants were regarded as having a poor outcome on all three measures. Objectively, a third of the sample were receiving financial support and their mood disorder was associated with 'vocational inactivity'; findings that parallel those reported for older adult populations. ${ }^{3,6,31,32}$ Subjectively, the cohort reported impaired role functioning for $25 \%$ of the past month (and were bed-ridden for about 5 days/month). Alonso and colleagues ${ }^{1}$ highlighted that, compared with other neurological and mental disorders, bipolar disorder was the most disabling disorder among adults because individuals showed role impairment for about 17 days per year. Even allowing for any overestimation of the level of impairment because of acute distress or current mood symptoms, it is clear that the young adults in this cohort are already at risk of equalling (or more likely exceeding) this predicted level of disability. Finally, the median score on the clinician-rated SOFAS assessment suggests our study population is equally or more impaired than other young adult populations with mood disorders recruited via community or clinical studies and/or older adults participating in studies undertaken across a wide range of settings. ${ }^{13-20,33}$

The logistic regression analyses demonstrate that the factors associated with impairment in young adults with mood disorders share many clinical features with older adults with unipolar and bipolar disorders. ${ }^{4-8,34}$ Similarities include the association with lifetime rates of anxiety disorders and substance use, especially cannabis, and the association with acute symptoms/distress. ${ }^{4-9,31,32}$ The findings undermine the assumption that significant impairments in functioning in mood disorders are a late consequence of the disorder and its treatment (such as those related to the adverse effects of multiple illness episodes, persistent subsyndromal symptoms, evolving complexity, comorbidities and/or polypharmacy). Our study highlights that gender, cannabis and nicotine use were more strongly related to impairment than diagnosis and it is clear that these associations warrant further consideration and explanation in prospective follow-up and case-control studies.

\section{Gender}

There was a statistical trend that reached significance in some analyses for female gender to be related to poorer functioning. In many studies of older adults, males are frequently reported to have worse outcomes than females, an association that is frequently assumed to be linked with the higher levels of alcohol and cannabis use in these populations. ${ }^{2-4}$ Alcohol use was not as critical in the youth cohort we studied, but frequent cannabis use was significantly more common in males (whether they had unipolar or bipolar disorder). More recently, studies using selfrated measures of quality of life identified poorer functioning in females, especially associated with behavioural and medical comorbidities. ${ }^{8,35}$ Although we did not include measures of physical health, other factors that influence functional outcomes,

Table 3 Multinomial logistic regression model showing factors associated with observer-rated social and occupational impairment

\begin{tabular}{|lccc|} 
& & \multicolumn{3}{c}{ OR $(95 \% \mathrm{Cl})$} \\
\cline { 2 - 4 } & Impaired $v$. mildly impaired & Moderately impaired $v$. mildly impaired & Severely impaired $v$. mildly impaired \\
\hline Kessler-10 score & $1.03(1.00-1.05)$ & $1.07(1.04-1.11)$ & $1.08(1.06-1.11)$ \\
\hline Any anxiety disorder & $2.09(1.17-3.73)$ & - & $5.85(1.98-17.27)$ \\
\hline Female gender & - & $1.36(1.05-1.96)$ & - \\
\hline Daily nicotine use & - & - & $1.21(1.05-1.37)$ \\
\hline $\begin{array}{l}\text { a. Defined by quartiles for Social and Occupational Functioning Assessment Scale (SOFAS) scores. The groups 'Impaired' (score 61-65), 'moderately impaired' (score 51-60) and } \\
\text { severely impaired' (score <51) are compared with the 'mildly impaired' reference group (score }>\text { 65). }\end{array}$
\end{tabular}


such as comorbid anxiety and current distress levels, were both significantly more common in females in our study. The crosssectional analysis does not allow us to determine cause and effect, so we could not examine whether distress undermines functioning more in females than males or whether poor functioning is more psychologically stressful to females.

\section{Cannabis and nicotine use}

We cannot disentangle whether the association between cannabis use and anxiety represents a direct or indirect effect, as it is not possible to determine from this data-set whether cannabis use is a method of self-medicating to reduce symptoms of anxiety or distress, or whether cannabis use impairs functioning and leads to more distress.

The repeated demonstration of an association between nicotine use and impairment and the size of the OR suggest this finding is unlikely to be the result of chance. It is conceivable that it represents an indirect association with or proxy measure of cannabis use (as there is a highly significant overlap between nicotine and cannabis use, with or without use of alcohol). Alternatively, it is frequently suggested that mood and substance use disorders share a number of risk factors, including genetic predisposition. ${ }^{36,37}$ We cannot test these models on the current data-set, but there is a growing body of research suggesting a complex but inconsistent, and/or bidirectional, relationship between smoking status and mood disorders, especially in those with an early age at onset. ${ }^{34,38-39}$ Nicotine use has been shown to be associated with greater symptom burden in mood disorders, higher levels of impairment, poorer treatment response and delayed recovery in clinical samples. However, lack of statistical power in those clinical studies may mask any gender differences. By contrast, large-scale community studies, such as Richardson et $a{ }^{40}{ }^{40}$ demonstrate gender-specific associations between mood disorders and nicotine use. Rates of mood disorder were increased in female smokers compared with non-smokers ( $\mathrm{OR} \sim 4$ ) and female, but not male, smokers showed statistically significant increases in the prevalence of severe mental disorders, subthreshold disorders, all mood and anxiety symptoms, and impairment, with the latter especially related to academic functioning.

\section{Limitations}

The current study has limitations that need highlighting. First, we did not have access to other clinical case record data on premorbid personality, physical disorders and/or specific details of current treatments (for example information about physical health and treatments was recorded in all clinical notes, but not included in all research databases) or the neurocognitive profile. Previous studies of functioning in young people with mood disorders have not routinely reported data on medical comorbidities, ${ }^{17-19,31}$ and although type of treatment was not shown to have any influence on functioning in young people with unipolar or bipolar disorder in a recent study, ${ }^{16}$ these factors do need to be assessed in future research. It is known that medical disorders are associated with worse functioning in older adults with mood disorders and both side-effects of medication and neurocognitive profile have been implicated in some but not all previous studies on functioning in mood disorders. ${ }^{1-5,32,33}$ The lack of a structured personality assessment represents a pragmatic decision related to the wish to maximise study recruitment by minimising the burden placed on study participants and the time allocated to research ratings (such as a personality assessment interview). Plus, as there is uncertainty about the reliability and validity of an adult personality assessment schedule when applied to youth with a current mood episode, an informed decision to exclude this from the protocol was made.

Finally, we did not use a precise rating of manic or depressive symptoms. The former was excluded as it is established that impaired functioning, even in bipolar I disorder, is primarily related to depressive symptoms or other factors, with the manic symptoms accounting for only a small proportion of the explained variance in impairment and having poor predictive validity. ${ }^{5,6,8,9,16,17}$ We also preferred the $\mathrm{K} 10$ as it rates the impact of all the symptoms experienced, including comorbid disorders, rather than focusing exclusively on depressive symptoms, as the latter would capture only one aspect of the clinical presentation of mood disorders in younger adults. Employing the K10 allowed us to take into account the association between functioning and current distress without focusing speciously on symptoms related to one diagnostic category. ${ }^{23-26}$

\section{Implications}

In summary, this large-scale study in young adults with mood disorders demonstrates that functional impairment is marked even in these early stages of illness. Comorbid disorders and substance use are ubiquitous, undermining the utility of measuring episode outcomes by rating mood symptoms alone. In future, focusing outcome measures on functioning may provide more meaningful outcome assessments. Furthermore, such ratings are applicable to any age or diagnostic subgroup, as functional recovery is usually associated with syndromal recovery, whereas significant clinical improvement is not necessarily associated with functional recovery.

Jan Scott, MBBS, MD, FRCPsych, Academic Psychiatry, Institute of Neuroscience, Newcastle University, Newcastle and Centre for Affective Disorders, Institute of Psychiatry, London, UK; Elizabeth M. Scott, MBChB, FRANZCP, Clinical Research Unit, Brain \& Mind Research Institute, University of Sydney and School of Medicine, , Django Whit Bradley Whitwell, DipAppSci, Jim Lagopoulos, MBiomedE, PhD, FAINM, Ian B. Hickie, MD, FRANZCP, Clinical Research Unit, Brain \& Mind Research Institute, University of Sydney, Sydney, Australia

Correspondence: Jan Scott, Newcastle University, Academic Psychiatry, Newcastle General Hospital, Westgate Road, Newcastle NE4 6BE, UK. Email: jan.scott@newcastle.ac.uk

First received 24 Jun 2013, final revision 17 Mar 2014, accepted 28 Mar 2014

\section{Funding}

I.B.H., D.F.H. and A.J.G. were supported by an NHMRC Australia Fellowship awarded to I.B.H. (No. 464914). S.L.N. was funded by an NHMRC Clinical Research Fellowship (No. 402864). This research was further supported by NHMRC Program Grant (No. 566529) and Centres of Clinical Research Excellence Grant (No. 264611). D.F.H. was supported by a grant from the NSW Health, Mental Health and Drug \& Alcohol Office. These funding agencies had no further role in study design, in the collection, analysis and interpretation of cata; in the writing of the report; and in the clecision to submit the paper for publication. J.S. recived an It that funded a secondment to the Brain and Mind Research Institute; which enabled her to participate in this and a number of other research projects and to co-author publications. Her current research on young people at risk of mood disorders is supported by an NHS RfPb grant (PB-PG-0609-16166).

\section{References}

1 Alonso J, Petukhova M, Vilagut G, Chatterji S, Heeringa S, Üstün TB, et al. Days out of role due to common physical and mental conditions: results from the WHO World Mental Health surveys. Mol Psychiatry 2011; 16: 1234-46.

2 Dean BB, Gerner D, Gerner RH. A systematic review evaluating health-related quality of life, work impairment, and healthcare costs and utilization in bipolar disorder. Curr Med Res Opin 2004; 20: 139-54.

3 Gilbert E, Marwaha S. Predictors of employment in bipolar disorder: a systematic review. J Affect Disord 2013; 145: 156-64. 
4 Grande I, Goikolea JM, de Dios C, González-Pinto A, Montes JM, Saiz-Ruiz J, et al. Occupational disability in bipolar disorder: analysis of predictors of being on severe disablement benefit (PREBIS study data). Acta Psychiat Scand 2013; 127: 403-11.

5 Judd LL, Schettler PJ, Solomon DA, Maser JD, Coryell W, Endicott J, Akiskal HS. Psychosocial disability and work role function compared across the long-term course of bipolar I, bipolar II and unipolar major depressive disorders. J Affect Disord 2008; 108: 49-58.

6 Kennedy N, Foy K, Sherazi R, McDonough M, McKeon P. Long-term social functioning after depression treated by psychiatrists: a review. Bipolar Disord 2007; 9: 25-37.

7 Kessler RC, Akiskal HS, Ames M, Birnbaum H, Greenberg P, Hirschfeld RM, et al. Prevalence and effects of mood disorders on work performance in a nationally representative sample of U.S. workers. Am J Psychiatry 2006; 163 $1561-8$

8 Morriss R, Yang M, Chopra A, Bentall R, Paykel E, Scott J. Differential effects of depression and mania symptoms on social adjustment: prospective study in bipolar disorder. Bipolar Disord 2013; 15: 80-91.

9 Simon GE, Bauer MS, Ludman EJ, Operskalski BH, Unützer J. Mood symptoms, functional impairment, and disability in people with bipolar disorder: specific effects of mania and depression. J Clin Psychiatry 2007; 68: $1237-45$.

10 Balazs J, Miklosi M, Kereszteny A, Hoven CW, Carli V, Wasserman C, et al. Adolescent subthreshold-depression and anxiety: psychopathology, functional impairment and increased suicide risk. J Child Psychol Psychiatry 2013; 54: 670-7.

11 McGorry PD, Hickie IB, Yung AR, Pantelis C, Jackson HJ. Clinical staging of psychiatric disorders: a heuristic framework for choosing earlier, safer and more effective interventions. Aust NZ J Psychiatry 2006; 40: 616-22.

12 Scott EM, Hermens DF, Glozier N, Naismith SL, Guastella AJ, Hickie IB. Targeted primary care-based mental health services for young Australians. Med J Aust 2012; 196: 136-40.

13 Scott J, Leboyer M, Hickie I, Berk M, Kapczinski F, Frank E, et al. Clinical staging in psychiatry: a cross-cutting model of diagnosis with heuristic and practical value. Br J Psychiatry 2013; 202: 243-5.

14 Derdikman-Eiron R, Indredavik MS, Bakken IJ, Bratberg GH, Hjemdal O, Colton M. Gender differences in psychosocial functioning of adolescents with symptoms of anxiety and depression: Iongitudinal findings from the Nord-Trondelag Health Study. Soc Psychiatry Psychiatr Epidemiol 2012; 47 1855-63.

15 Goldstein TR, Birmaher B, Axelson D, Goldstein BI, Gill MK, Esposito-Smythers C, et al. Psychosocial functioning among bipolar youth. J Affect Disord 2009; 114: $174-83$.

16 Jansen K, Magalhaes PV, Tavares Pinheiro R, Kapczinski F, Silva RA. Early functional impairment in bipolar youth: a nested population-based case-control study. J Affect Disord 2012; 142: 208-12.

17 Kauer-Sant'Anna M, Bond DJ, Lam RW, Yatham LN. Functional outcomes in first-episode patients with bipolar disorder: a prospective study from the Systematic Treatment Optimization Program for Early Mania project. Compr Psychiatry 2009; 50: 1-8.

18 Lewinsohn PM, Klein DN, Seeley JR. Bipolar disorders in a community sample of older adolescents: prevalence, phenomenology, comorbidity, and course. J Am Acad Child Adolesc Psychiatry 1995; 34: 454-63.

19 Rosa AR, Gonzalez-Ortega I, Gonzalez-Pinto A, Echebura E, Comes M, Martinez-Àran A, et al. One-year psychosocial functioning in patients in the early vs. late stage of bipolar disorder. Acta Psychiatr Scand 2012; 125: 335-41.

20 Scott EM, Hermens DF, Naismith SL, Guastella AJ, De Regt T, White D, et al Distinguishing young people with emerging bipolar disorders from those with unipolar depression. J Affect Disord 2013; 144: 208-15.
21 American Psychiatric Association. Diagnostic and Statistical Manual of Mental Disorders (4th edn, revised) (DSM-IV-TR). APA, 2000.

22 Hickie IB, Scott EM, Hermens DF, Naismith SL, Guastella AJ, Kaur M, et al. Applying clinical staging to young people who present for mental health care. Early Interv Psychiatry 2013; 7: 31-43.

23 Humeniuk R, Ali R, Babor TF, Farrell M, Formigoni ML, Jittiwutikarn J, et al. Validation of the Alcohol, Smoking And Substance Involvement Screening Test (ASSIST). Addiction 2008; 103: 1039-47.

24 Kessler RC, Andrews G, Colpe LJ, Hiripi E, Mroczek DK, Normand SL, et al. Short screening scales to monitor population prevalences and trends in non-specific psychological distress. Psychol Med 2002; 32: 959-76.

25 Andrews G, Slade T. Interpreting scores on the Kessler Psychological Distress Scale (K10). Aust N Z J Public Health 2001; 25: 494-7.

26 Angst J, Gamma A, Clarke D, Ajdacic-Gross V, Rossler W, Regier D. Subjective distress predicts treatment seeking for depression, bipolar, anxiety, panic, neurasthenia and insomnia severity spectra. Acta Psychiatr Scand 2010; 122: 488-98.

27 Mojtabai R, Olfson M, Mechanic D. Perceived need and help-seeking in adults with mood, anxiety, or substance use disorders. Arch Gen Psychiatry 2002; 59: 77-84.

28 Von Korff M, Ustun TB, Ormel J, Kaplan I, Simon GE. Self-report disability in an international primary care study of psychological illness. J Clin Epidemiol 1996; 49: 297-303.

29 Goldman $\mathrm{HH}$, Skodol AE, Lave TR. Revising axis $\mathrm{V}$ for DSM-IV: a review of measures of social functioning. Am J Psychiatry 1992; 149: 1148-56.

30 Australian Institute of Health \& Welfare. Young Australian Health and Well-being Survey of 2011: 201. Australian Institute of Health \& Welfare.

31 Isometsa ET, Katila $\mathrm{H}$, Aro T. Disability pension for major depression in Finland. Am J Psychiatry 2000; 157: 1869-72.

32 Waghorn G, Chant D, Jaeger J. Employment functioning and disability among community residents with bipolar affective disorder: results from an Australian community survey. Bipolar Disord 2007; 9: 166-82.

33 Karlsson L, Pelkonen M, Heilä $\mathrm{H}$, Holi M, Kiviruusu O, Tuisku V, et al. Differences in the clinical characteristics of adolescent depressive disorders. Depress Anxiety 2007; 24: 421-32.

34 Lev-Ran S, Le Foll B, McKenzie K, George TP, Rehm J. Bipolar disorder and co-occurring cannabis use disorders: characteristics, co-morbidities and clinical correlates. Psychiatry Res 2013; 209: 459-65.

35 de la Cruz MS, Lai Z, Goodrich DE, Kilbourne AM. Gender differences in health-related quality of life in patients with bipolar disorder. Arch Womens Ment Health 2013; 16: 317-23.

36 Holma IA, Holma KM, Melartin TK, Ketokivi M, Isometsä E. Depression and smoking: a 5-year prospective study of patients with major depressive disorder. Depress Anxiety 2013; 30: 580-8.

37 Dierker LC, Avenevoli S, Stolar M, Merikangas KR. Smoking and depression: an examination of mechanisms of comorbidity. Am J Psychiatry 2002; 159: 947-53.

38 Grant BF, Stinson FS, Dawson DA, Chou SP, Dufour MC, Compton W, et al Prevalence and co-occurrence of substance use disorders and independent mood and anxiety disorders: results from the National Epidemiologic Survey on Alcohol and Related Conditions. Arch Gen Psychiatry 2004; 61: 807-16.

39 Dierker L, Donny E. The role of psychiatric disorders in the relationship between cigarette smoking and DSM-IV nicotine dependence among young adults. Nicotine Tob Res 2008; 10: 439-46.

40 Richardson A, He JP, Curry L, Merikangas K. Cigarette smoking and mood disorders in U.S. adolescents: sex-specific associations with symptoms, diagnoses, impairment and health services use. J Psychosom Res 2012; 72: $269-75$ 